\title{
Penser la co-activité maître-élèves comme une nouvelle expertise enseignante
}

L'émergence du concept d'auteur dans la classe : une construction sémiotique complexe

\section{Alain Decron}

\section{OpenEdition}

\section{Journals}

Édition électronique

URL : http://journals.openedition.org/trema/820

DOI : 10.4000/trema.820

ISSN : 2107-0997

Éditeur

Faculté d'Éducation de l'université de Montpellier

Édition imprimée

Date de publication : 1 octobre 2005

Pagination : 145-157

ISSN : 1167-315X

Référence électronique

Alain Decron, "Penser la co-activité maître-élèves comme une nouvelle expertise enseignante », Tréma [En ligne], 24 | 2005, mis en ligne le 29 septembre 2010, consulté le 19 avril 2019. URL : http:// journals.openedition.org/trema/820; DOI : 10.4000/trema.820

Ce document a été généré automatiquement le 19 avril 2019

Trema 


\section{Penser la co-activité maître-élèves comme une nouvelle expertise enseignante}

L'émergence du concept d'auteur dans la classe : une construction sémiotique complexe

\section{Alain Decron}

Nous comprenons enfin que le récit est une affaire sérieuse, que ce soit dans le domaine de la loi, de la littérature ou de la vie. Mais ce n'est pas tout. Il n'est sans doute pas d'activité de l'esprit qui procure de tels délices, tout en faisant courir de tels périls. Jérôme BRUNER 
1 Cette contribution a pour objet de mettre en travail une problématique qui se nourrit à la fois d'un questionnement lié à la pratique de terrain et d'une recherche en cours au sein de deux équipes: le LIRDEF (IUFM Montpellier), dirigée par Marc Durand, et plus spécialement sa composante Alfa sous la responsabilité de Dominique BUCHETON, l'IRSCE (EA n 763 Université de Perpignan) dirigée par Joëlle RETHORE. Une communication de ce travail a été faite lors du Colloque de Bordeaux en avril 2003.

2 Nous cherchons à savoir comment se construit dans la co-activité langagière et cognitive maître-élèves, la bascule enseignement-apprentissage. Nous

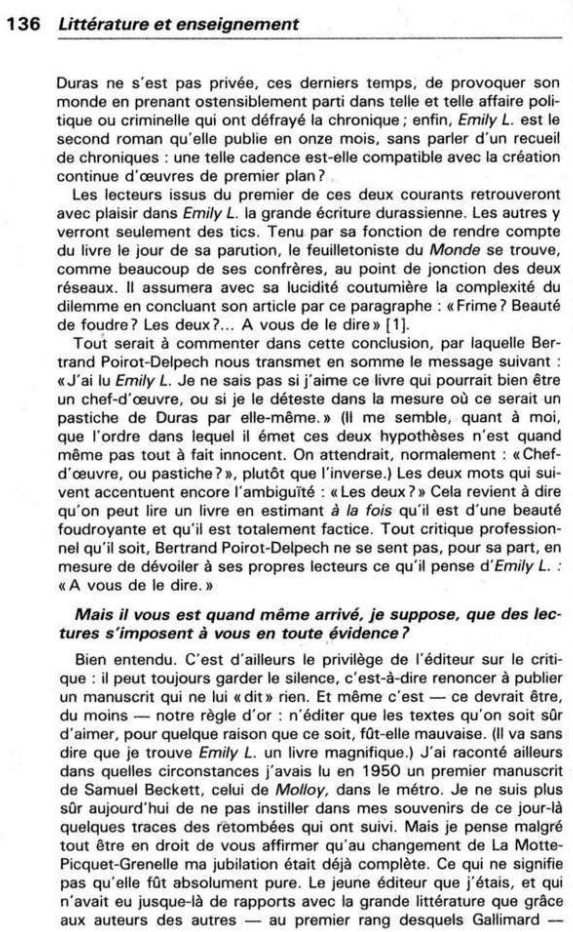
émettons l'hypothèse que cette bascule se produit grâce à la circulation des sujets de la classe au sein de trois univers discursifs, circulation qui permet à chacun d'investir la puissance du langage qu'il a expérimentée depuis son plus jeune âge (depuis sa conception, dirait Françoise Dolto) comme interprète et qui a produit en lui des effets sur les plans cognitif et affectif. Nous faisons également l'hypothèse que l'Ecole primaire française ne permet pas nécessairement aux élèves cette circulation, d'où l'intérêt de la théoriser pour que chaque enseignant puisse en faire un élément de sa praxis.

3 Après un travail oral sur l'album Yakouba, KhadijaB, élève de CM2 en ZEP, écrit sur son cahier de travail :

Pour être un guerrier et avoir du courage, il ne suffit pas de tuer un lion, il faut aussi avoir du cœur et refuser certaines traditions.

Elle produit là un langage de grande qualité qui à la fois résume l'intrigue de l'histoire, donne sa position éthique, présente les grands enjeux soulevés par ce récit : l'acceptation ou le refus de la tradition.

5 La question est : comment KhadijaB arrive-t-elle à une telle puissance langagière ?

6 Que se passe-t-il dans cette classe qui semble accompagner les élèves vers une élaboration cognitive et langagière d'une telle ampleur?

7 Nous allons revenir sur des productions de cette classe grâce aux extraits de corpus qui éclaireront notre réflexion d'une dimension pratique. 
pensée de chacun des protagonistes et permettre son développement dans l'échange. Nous poserons même la classe comme une construction langagière entretenant un rapport dialectique entre expression du «déjà connu» et enquête permanente pour construire de la signification, et donc d'une certaine façon intégrer, incorporer de l'inconnu. BRUNER (2002) montre combien tout est récit en s'appuyant à la fois sur des récits juridiques, littéraires et des récits de vie. Tout est récit car dès que nous prenons la parole pour re-présenter le monde, nous effectuons des choix, des focalisations, nous employons des péripéties, nous réorganisons le substrat perceptif et affectif pour en faire l'objet de la mise en mot. Mais ces mots, la prosodie, les événements mimogestuels accompagnateurs des seuls signes linguistiques, l'organisation bidimensionnelle de la page écrite, ne sont pas le monde. Ils sont notre représentation du monde filtrée par notre point de vue singulier de sujet «historicisé », contraint par un code arbitraire et conventionnel, pour signifier et proposer ou forcer l'interprétation par soi-même et par les autres. Dire le monde revient à revendiquer une place d'auteur à la source d'une communauté sémiotique (DECRON, 2001, p. 10), capable d'amener de la surprise (BRUNER, 2002, pp. 8-9, 16, 28, 66) et de tisser des voix ou encore clore l'univers de la fiction (BAKHTINE, 1984).

9 Nous pourrions alors émettre l'hypothèse que la classe est un texte, au sens où DUCROT l'écrit : «Certains enfin lui donnent [au texte] une extension trans-sémiotique, parlant de texte filmique, texte musical, etc. ». La classe deviendrait alors une « chaîne langagière et non simplement linguistique - formant une unité communicationnelle». Nous rajouterons alors « une unité communicationnelle et cognitive » pour insister sur cette double circulation langage-pensée inhérente à toute production langagière.

Si l'on admet que la classe est un texte tissé d'actes d'enseignement et d'actes d'apprentissages, nous devons questionner à la fois les champs théorique et pratique pour tenter de comprendre les enjeux cognitifs, langagiers et identitaires de ces échanges.

11 Qu'est-ce qui dans la pédagogie employée facilite ou empêche des prises de parole que nous ressentons comme essentielles? Qu'est-ce qui installe la réflexivité dans une activité ? Comment initier une circulation cognitivo-langagière plutôt que de créer des figements? Ne devons-nous pas tenter de définir une nouvelle professionnalité, une nouvelle expertise du maitre?

\section{Tous les acteurs de l'Ecole sont des sujets en construction}

Tous les auteurs auxquels nous nous référons ont une caractéristique commune, c'est la place centrale qu'ils donnent au sujet en train de se construire (BAUTIER et BUCHETON, 1995 ; BAUTIER et ROCHEX, 1993; ROCHEX, 2002). Ce sujet tisse des discours multiples (BAKHTINE, 1984; BRES, 1999), circule sur des postures différentes (BUCHETON, 1998, 2000), met sa vie en récit (Bruner, 2002) pour trouver un tuteur de résilience (CYRULNIK, 2003). Tous ses discours sont plongés dans son histoire, ses répertoires privés (PEIRCE, 1978; RETHORE, 1998), ce qui lui permet un accès singulier à la culture (BRUNER, 1991, 2002), un point de vue singulier sur le monde (BUCHETON, 1995, 2000). Ce sujet est inscrit au sein de la chaîne des interprétants (PEIRCE, 1978) et contribue donc à élaborer de la signification en «métabolisant» les signes qui le traversent, dans un tissage serré de commun et d'individuel (FRANÇOIS, 2002).

Tréma, 24 | 2005 
13 La pensée et le langage se construisent en synergie, au cœur d'interactions sociales (VYGOTSKI, 1934/1997). Cette co-construction mène vers des apprentissages grâce à l'étayage (VYGOTSKI, 1934/1997 ; BRUNER, 1991 et 1997) de référents qui réajustent en permanence leurs interventions en fonction de leur évaluation des capacités comportementales de l'apprenant (BRONCKART, 2002).

Ces apprentissages nécessitent que la parole s'inscrive (BALAT, 2000), ait donc une portée perlocutoire et autoperlocutoire (AUSTIN, 1970). Ne retrouvons-nous pas alors cette question de l'auteur scolaire (DECRON, 2001) qui assume une parole ouverte sur l'interprétation?

\section{La classe est un lieu où l'on se raconte des histoires}

La classe est une production langagière éminemment dialogique (BAKHTINE, 1984). Dialogique car s'entrecroisent sans cesse des discours nourris par d'autres discours (BRES, 1999) : le discours des Instructions Officielles, des parents, des collègues, des camarades, des acteurs sociaux, de la télé, des journaux, des politiques, des chercheurs, de l'IUFM, de la hiérarchie, de la doxa, le discours fantasmé que l'on pense que l'autre pourrait tenir... Nous sommes donc en permanence à la croisée de discours et d'énonciateurs présents ou non qui présentent ou représentent en classe le monde et ses événements.

Pour BRUNER (2002, p. 47), le récit «subjonctivise » la réalité : il la subvertit et la rend éclatante à la fois. Le récit qui opère sans cesse des choix conscients ou inconscients donne sa place à ce qui a été mais ouvre aussi des champs du possible.

Le récit n'est pas un retour du passé, comme le dit CYRULNIK (2003, p. 129), mais une façon de faire signe avec son passé, l'interpréter (PEIRCE, 1978), le remanier et le présenter grâce à des choix énoncés dans le flux continu de l'oral ou dans la bidimensionnalité de l'écriture, en tous cas avec une importante force d'abstraction.

18 Le récit pratique donc l'irruption de l'inattendu, de la surprise, de l'inconnu dans le familier et le déjà su. BRUNER reprend d'ailleurs le mot de peripéteia $(2002$, p. 8) qu'il emprunte à ARISTOTE. Cette irruption, cette péripétie, sont à l'origine d'une inscription symbolique, puisque CYRULNIK (2003, p. 22) affirme que «quelque chose dans le réel doit provoquer une surprise et une signification qui rendent la chose saillante" pour qu'il y ait événement, et que «CET EVENEMENT SOIT EXCITANT » rajoute BRUNER (2002, p. 47).

Nous voyons donc l'importance du surgissement de la surprise pour pratiquer une rupture avec ce qui est familier. C'est une façon de rejoindre VYGOTSKI (1934 / 1997) lorsqu'il énonce son concept de «zone prochaine de développement» (pp. 351-353). En effet : «L'APPRENTISSAGE N'EST VALABLE QUE S'IL DEVANCE LE DEVELOPPEMENT. » écrit-il, page 358. C'est grâce à cette rupture qu'apparaît l'événement en tant que tel et donc susceptible de marquer l'esprit du sujet. La fonction intégrative, d'après les trois auteurs déjà cités, sera tenue par le langage produit autour de l'événement. Langage émis par d'autres et par soi-même, y compris en cas de monologue intérieur. Le récit permet de domestiquer l'erreur et la surprise écrit BRUNER (2002, p. 30), car nous avons de grands 
«plans» en tête qui nous incitent à prévoir le possible, alors que c'est un confort irréaliste, avertit BALAT.

Une question se pose alors : quel est le langage produit? Certaines formes ou genres sontils plus performants? Les assertions réalisées nous renseignent-elles sur la position cognitive, psychologique du sujet ainsi que sa capacité actuelle à incorporer un savoir ou le transmettre?

BRUNER (2002, p. 18) insiste sur cette possibilité de généralisation: "C'est en effet la traduction dans les conventions du récit qui permet de convertir l'expérience individuelle en une monnaie collective [...]». L'aspect conventionnel est à la base d'une intercompréhension, comme c'est le cas pour le signe linguistique, mais comment, dans le cadre d'un récit cela est-il possible ? L'accès à une culture commune de mythes, contes, histoires et légendes, s'il est nécessaire, est-il suffisant? Comment les images mentales d'un locuteur produisent-elles des images mentales chez l'autre? Comment passe-t-on de l'intrapersonnel à l'interpersonnel et au collectif? Nous sommes là au cœur de la bascule enseignement-apprentissages et il semble nécessaire d'interroger et penser plus loin la Zone Proximale de Développement (ZPD) de VYGOTSKI.

2 Autrement dit, si l'on se réfère à Bruner (2002, pp. 26-27) à quelle(s) condition(s) la métaphore est-elle vécue comme une métaphore, avec toute sa puissance d'élévation audessus des détails?

Pour BRONCKART (2002, p. 36) reprenant VYGOTSKI, le discours littéraire opère une transformation de la vie psychique de son récepteur grâce à la valeur pragmatique des textes littéraires et au caractère doublement dialectique des processus en jeu dans leur production-interprétation.

24 Nous ne pouvons faire abstraction du fait que le récit est également fortement lié à l'acte thérapeutique : pensons à la consultation chez le médecin, mais surtout à la psychanalyse. L'Ecole de Palo Alto, à l'origine des thérapies brèves, a permis à de nombreux chercheurs d'expérimenter des protocoles de prise en charge très différents de la psychanalyse. ERICKSON, en particulier a travaillé sur l'hypnose et le récit comme vecteur thérapeutique : il racontait des histoires à haute portée métaphorique à ses patients pour induire un changement de point de vue et de comportement.

Dans le cadre de l'enseignement, et plus précisément de l'AIS, le maitre G travaille continuellement sur le récit littéraire, le récit de vie, pour tisser des liens, subjonctiviser le monde (BRUNER, 2002, pp. 14-15), devenir un tuteur de résilience (CYRULNIK, 2003, pp. 98-99 et 176-177). Ne devrait-on pas considérer ce travail sur la mise en récit du monde, comme faisant partie intégrante d'une nouvelle professionnalité pour agir en amont de la réorientation, c'est-à-dire quand le geste est encore éducatif, avant de verser dans la thérapeutique?

\section{Le sujet se construit par étapes intermédiaires}

Dominique BUCHETON (1995) puis Dominique BUCHETON et Jean-Charles CHABANNE (2002) décrivent et précisent le concept d'écriture-réécriture. Dans les pratiques sociales, les textes d'écrivains, journalistes et autres, sont réécrits plusieurs fois avant d'aboutir à un «jet» socialisable. Claudine FABRE (1990) nous a montré combien le « brouillonnement » est indispensable à l'écriture des écoliers. Il semble donc nécessaire 
de penser en termes d'étapes intermédiaires du dire-penser. Nous sommes d'ailleurs confortés dans cette thèse par l'apport de BRUNER (2002, pp.58-59) pour qui nous passons notre temps à « construire et reconstruire notre Moi » à l'aide « d'histoires qui s'accumulent au fil des années».

«Parler et plus encore écrire, c'est projeter une image de soi. C'est donc prendre le risque de se constituer en personne " écrivent Jean-Charles CHABANNE et Dominique BUCHETON (2002, p. 12). Se constituer en personne, c'est faire sonner la parole à travers soi, c'est-à-dire au filtre de son Moi. Le lien se noue entre étapes intermédiaires de l'écriture, et étapes intermédiaires de la construction du sujet. Chacun, au sein de la chaîne des interprétants (PEIRCE, 1978, 1993, 1998), va faire signe avec un discours et le sémiotiser. La prise de risque réside dans le dévoilement partiel qui régit la manière de faire signe avec un autre discours, ou de laisser entrevoir son Moi à la communauté. Mais cette prise de risque fait grandir, changer, permet de se construire par un jeu de miroir, grâce à la reconnaissance des autres.

Les productions langagières intermédiaires sont donc intermédiaires à de multiples niveaux, comme le montrent CHABANNE et BUCHETON (2002, p. 26). Par sa confrontation avec d'autres productions issues de soi-même ou d'autres que soi, en synchronie et diachronie, le locuteur se perçoit comme un sujet culturel, historicisé et unique. Culturel, car tout discours est situé, y compris au sein de l'Ecole qui installe des rites et des genres culturels. Historicisé car le sujet prend alors conscience de son évolution au fil du temps et suivant les contextes (un même objet de savoir n'est plus éclairé, filtré, regardé de la même façon). Unique car le locuteur affirme un point de vue, le confronte aux autres et peut mesurer les écarts, se déplacer sans se perdre, dans un jeu de tension entre socialisation et individuation. «Se construire, n'est-ce pas après tout la seule façon d'établir que nous sommes uniques. [...] C'est en comparant les récits que nous produisons sur nous-mêmes avec ceux que les autres nous proposent d'eux-mêmes que nous y parvenons » écrit BRUNER (2002, p. 59).

BERNIE (2001, p. 157) écrit: "[...] dans les situations scolaires, l'énonciateur est invité à se construire dans de nouvelles sphères d'échanges à la fois comme sujet social et comme sujet cognitif, en un seul et même mouvement de construction de nouveaux contextes discursifs et d'appropriation de nouveaux contenus. Il est admis, bien qu'encore peu démontré, que la traversée de plusieurs contextes participe du processus de décontextualisation / recontextualisation des significations indispensable à leur conceptualisation. »

31 Cette traversée oblige à créer des étapes didactiques pour faciliter des variations et des déplacements cognitivolangagiers chez les élèves.

\section{Langage et pensée s'interfécondent dans des logiques de positionnement au monde différentes}

PEIRCE (1978) définit, dans sa phénoménologie ou phanéroscopie, trois catégories qui suffisent à décrire les phénomènes du monde : la priméité, la secondéité, la tiercéité.

Essayons brièvement de comprendre ce que recouvrent ces trois catégories avec Michel BALAT (2000) :

- La priméité est l'univers des qualités. Chaque qualité est ce qu'elle est, immuable, éternelle. 
- La secondéité est l'univers des existants, des rencontres, des résistances, des occurrences des phénomènes.

- La tiercéité est l'univers des lois, des règles, de la pensée. La pensée n'est pas immuable et peut croître, ce n'est donc pas une qualité. d'une telle approche théorique et phénoménologique pour mieux lire ou entendre les productions des apprenants, et par-là obtenir des indices intéressants sur leur travail cognitif.

41 Nous allons donc ouvrir une réflexion afin de nous donner quelques concepts intermédiaires pour analyser les interactions en classe, la co-activité maître-élèves. Nous questionnerons en particulier la tension circulation-figement au sein des échanges, car le langage nous permet d'observer les déplacements linguistiques, témoins de façons d'être au monde différentes, de "rapports à ", de positions cognitives et communicatives distinctes. Nous sommes d'ailleurs, en tant que chercheurs, dans des étapes intermédiaires du dire-penser pour comprendre comment une pensée crée une autre pensée, comment certaines formes d'étayage enclenchent le processus de signification, comment l'auteur investit sa position grâce à la visée universelle de ses propos.

42 Avec quels outils pouvons-nous écouter ou lire des productions discursives? Nous proposons quelques axes, quelques pistes semblant faciliter la compréhension des enjeux à la fois langagiers et cognitifs qui sous-tendent les séquences d'apprentissage.

\section{La signification se construit à plusieurs voix, dans la co-activité}

43 La différence fondamentale entre un discours d'adulte cultivé et celui d'un élève de l'école primaire réside dans la multi-référencialité du premier. L'adulte a intégré un certain nombre de voix qui se tissent pour éclairer un objet de savoir. Alors que les enfants petits, n'ont le plus souvent qu'une voix à leur disposition dans un moment 
donné : leur propre croyance, le discours du père, du maître, de la télé ou d'un autre référent crédible. Le rôle de la classe est de rendre visible ou audible chacune de ces voix singulières pour tisser ainsi un texte complexe, multiréférencé, polyphonique autour de l'objet en question. La classe deviendrait alors la représentation « dépliée » de l'objectif à atteindre : la constitution et la prise de conscience d'une groupalité interne (j'emprunte cette expression à la psychanalyse) ou un dialogisme intra-discursif intra-personnel si l'on se réfère à BAKHTINE.

La classe est un texte composé de la multiplicité et de l'hétérogénéité des propositions, des points de vue, qui s'organisent pour élaborer de la signification. Elle joue le rôle d'un prisme qui recompose la lumière à partir des rayons lumineux de longueurs d'ondes différentes. C'est dans les reprises lexicales, les procédés de nomination, les citations, que le discours sort du chaos pour prendre sens.

Nous allons retranscrire quelques extraits d'un débat oral.

Sofia : pendant qu'on lisait vous avez écrit deux consignes

Maître : oui /j'ai écrit deux consignes / oui

Sofia : écrire les idées fortes et que / et écrire dans le cahier de travail deux questions

Maître : deux questions

Sofia : deux questions qui nous posent problème

Maître : deux questions qui posaient problème / et alors on a essayé aussi de savoir euh ce que ça voulait dire / poser problème [...]

Maître : et quand on a tout compris on s'en pose pas des questions

KhadijaB : c'est des questions qui nous font réfléchir

Maître : ah / on peut quand même se poser des questions qui nous font réfléchir

Dans cet extrait, trois voix polyloguent pour réfléchir, penser ensemble l'utilité de se questionner sur un livre, même s'il semble facile d'accès dès la première approche. Les informations sont partagées entre les trois interlocuteurs qui tissent ensemble un texte, épaississent son sens, tout en précisant la tâche à accomplir et les postures d'activité attendues.

\section{Créer la surprise, amener l'inconnu, introduire des péripéties pour susciter la reprise du processus cognitif. Qui s'en charge?}

Si nous admettons que le récit est au fondement de notre manière de penser le monde (BRUNER, 2002), la classe est aussi un récit. Ce récit, nous l'avons dit est forcément chaotique et son organisation est une des fonctions de l'école. Nous devons au milieu de ce chaos prélever de l'inconnu pour en faire des objets compréhensibles, incorporables. Lorsque l'un des acteurs de la classe amène de la surprise, il met les autres en rapport avec un horizon de savoir non encore intégré, mais dont il pressent qu'il pourra faire sens grâce au travail de réflexion du groupe, des pairs. L'enquête initiée par la surprise se matérialise par les "traînées de parole » qu'elle suscite et qui correspondent à l'acceptation par d'autres locuteurs d'abandonner un instant ou mettre entre parenthèses leurs points de vue pour collaborer avec l'auteur du propos. Il est intéressant d'observer qui se charge de ces apports: le maître seulement? Des élèves? Les deux parties en alternance? C'est un indicateur de l'espace d'autorisation, du degré d'invention souhaité et de la capacité d'improvisation de l'enseignant. 
Miloud : euh / parce que quand quand on le lit vite / on va pas bien comprendre / et vous vous l'avez lu avec les virgules et tout / euh on l'a on l'a bien on l'a plus compris que quand on lit vite [...]

Maître : tiens je vais te poser une question chut non Thomas tais-toi tais-toi / / / on va aller plus loin dans la discussion / on va changer un petit peu euh / vous allez vous parler vous / on va parler de vous un petit peu / / / euh / si tu étais à la place de Yakouba / / / est-ce que tu aurais fait son choix /// de refuser la tradition [...]

Le maître ou un élève se charge d'introduire de l'inconnu, une péripétie dans le texte de la classe, ce qui entraîne non seulement une centration due à l'apparition d'un événement intellectuel, mais encore l'obligation de se déplacer dans une zone incertaine pour intégrer cet inconnu au déjà-là.

\section{Existe-t-il de l'indéterminé dans le discours ?}

49 Nous recherchons l'apparition d'un langage non encore formaté ou filtré par les exigences formelles scolaires. Les locuteurs qui le produisent sont dans un flux de pensée, parfois très flou, peu normé, proche du «brut de penser" selon l'expression de Dominique BUCHETON (1997). Le discours est souvent très peu indiciaire, et s'il l'est, ces indices ne sont pas forcément encore partageables par la communauté. La principale visée de ce langage est réflexive dans le sens où l'interprète ne peut encore clôturer. Le locuteur donne l'impression qu'il pose quelques éléments pour pouvoir penser plus loin, en interaction avec les autres. Il a besoin de temps, d'échanges et de réflexion pour travailler intellectuellement et langagièrement ces éléments cognitifs.

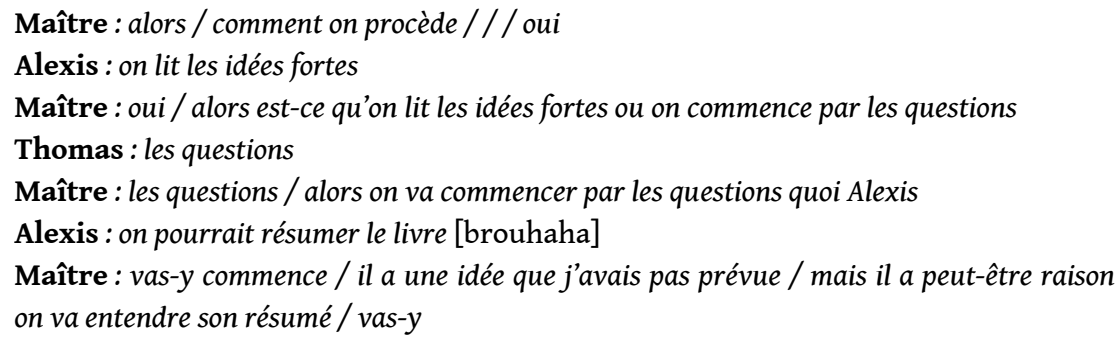

50 Nous avons un maître et des élèves qui pensent à haute voix, qui ne se figent pas sur une position arrêtée, un discours scolairement formaté lié à la préparation ou aux places de chacun. Cette part d'incertitude, de flou par rapport au discours habituel de l'institution laisse la place à l'interprétation. Tous se mettent à penser ce qui serait le plus intéressant pour débuter le cours. Tout en introduisant cette phase d'improvisation, le maitre signifie qu'il n'est pas dans la toute puissance, que des élèves peuvent s'impliquer eux aussi dans une pensée productive au niveau métacognitif. Cela entraine une forte adhésion de la classe derrière la proposition d'Alexis. Cette saynète marque le lien fort qui existe entre surprise, surgissement de l'inconnu, indétermination et ouverture de possibles.

\section{Existe-t-il de la précision dans le discours?}

51 Le discours se développe dans une visée à la fois explicative et communicative. Les locuteurs précisent, nomment, qualifient, définissent, d'une part pour faire correspondre le mieux possible le signe linguistique à l'objet «à dire », et d'autre part dans le but de mutualiser du savoir, de partager une culture par rapport à ce savoir. Le récit commence 
à se constituer ici, à partir de l'expérience personnelle, par l'affrontement, l'accord ou la synergie entre discours fortement indiciaires.

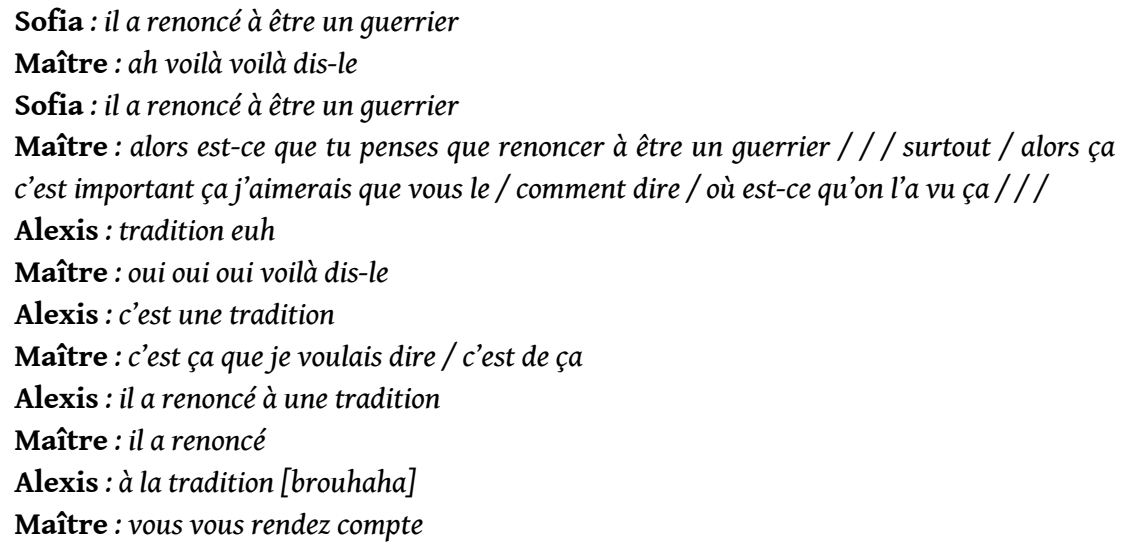

Dans cette saynète, nous avons un départ très indiciaire avec «renoncer ", « il » qui renvoie clairement à Yakouba et " guerrier » qui est un mot qui a fait l'objet de toute une discussion préalable pour en préciser le sens.

Mais ce qui est encore plus intéressant, puisque le travail de définition, de précision est un genre habituel de l'Ecole, c'est le glissement au cours du même échange vers une étape de généralisation qui arrive ici sous forme d'un hyperonyme " tradition » qui agit comme une nominalisation dialogique pour reprendre et contenir tous les tours de parole antérieurs. Ce faisant, le locuteur valide ce qui précède, signifie sa compréhension fine du concept et propose de le nommer.

Ce court extrait établit donc une transition avec le paragraphe suivant.

\section{Existe-t-il de la généralisation dans le discours ?}

L'auteur se donne les moyens cognitifs et langagiers de s'élever au-dessus des détails, de fonder une communauté sémiotique, de sortir de la simple confrontation d'existants, de points de vue singuliers, pour énoncer la loi, tendre vers l'universel. Le discours porte alors les traces linguistiques de la généralisation par hyperonymie, nomination dialogique, structure syntaxique même de l'énonciation.

Revenons à l'écriture de KhadijaB présentée au début de cet article. Quand elle écrit :

Pour être un guerrier et avoir du courage, il ne suffit pas de tuer un lion, il faut aussi avoir du coeur et refuser certaines traditions.

Elle emploie des formes verbales infinitives qui ont comme caractéristique de ne pas présenter de sujet grammatical réalisé. Chaque locuteur a donc la possibilité de s'insérer comme sujet possible de l'énonciation. Ces quelques lignes sonnent comme une morale de l'histoire, ont valeur de loi, pour se dégager des détails, en "faire une monnaie collective » comme l'écrit BRUNER (2002, p. 18).

\section{Circulation ou figement dans la co-activité ?}

Si nous admettons que le langage nous donne à voir les opérations mentales, le développement cognitif, les rapports au monde ou aux événements du monde, nous allons porter une attention particulière aux déplacements langagiers qui s'opèrent à 
l'intérieur d'une séance ou séquence d'apprentissage car ils signent les déplacements intellectuels des interlocuteurs. L'enquête pour élaborer de la signification à plusieurs serait à la fois une métaphore de la circulation interne à construire pour bénéficier d'un point de vue multi-référencé et l'indice d'une dynamique d'apprentissage.

La circulation permet en particulier de distinguer la construction d'une généralisation de la simple émission d'une généralité. C'est parce que le locuteur plonge dans ses répertoires privés, son flux intime de pensée, puis se confronte à d'autres pensées (internes ou externes), qu'il peut passer à l'énonciation de concepts établissant une passerelle cognitive entre individu et collectivité, entre expérience personnelle et savoir universel. La généralité ne consistant, elle, qu'en la reproduction d'une doxa sans nécessiter une implication ou une réflexion.

Alexis : bé euh / vu qu'il a pas tué le lion / pour ses proches c'est pas devenu un homme / alors que pour lui oui

Alexis est capable de prêter sa voix à deux points de vue différents et donc tenir un discours élaboré grâce à un déplacement de sa pensée sur d'autres raisonnements que le sien. C'est une trace de complexité du discours comme trace d'une complexité du «à dire ».

KhadijaA : tout à l'heure quand on on disait que que si on tuait pas le lion on on aurait la honte de sa famille / euh / / / certains hein je dis pas tout le monde mais certains se moquent de ce que pense la famille [brouhaha]

Maître : attends tais-toi

KhadijaA : j'ai dit certains se moquent de ce que pense la famille / / / euh comme Yakouba ///

Maître : lui se moque de ce que pense la famille

KhadijaA : non / euh / certains

Maître : ah euh approfondis un peu ton idée

KhadijaA : si moi j'aurais à affronter un lion pour être une guerrière / eh bé euh si par exemple il serait blessé et que je l'aurais pas tué mais que mes parents ma sœur bé la tradition tout ça eh bé ils auraient la honte de moi / bé je me moquerais de ce qu'ils pensent

Maître : oui par rapport à ce qu'on a dit /

KhadijaA : du moment que

Maitre: du moment que

KhadijaA : du moment que j'ai fait du bien pour le lion

61 Dans cet extrait, KhadijaA circule de l'indéterminé à la généralisation en passant par la précision. Indéterminé quand elle dit «certains", précision quand elle se réfère à "Yakouba " ou qu'elle féminise le mot "guerrière " pour le raccrocher à elle-même, généralisation quand elle affirme vouloir faire « du bien » à un lion générique qui n'est pas le même animal que celui rencontré par le guerrier africain.

KhadijaA nous montre donc une élaboration de la signification qui aboutit à un Dénivellement qui a pris racine à la fois dans de l'Indéterminé et de la Définition. C'est grâce à cette circulation, parfois implicite, parfois explicite, que beaucoup d'élèves, comme KhadijaA ou KhadijaB incorporent des savoirs savants sur la littérature, la langue en tant que système linguistique complexe, et des savoirs identitaires pour se situer dans le monde, continuer à le penser et s'y construire. 

six concepts intermédiaires nous invitent à élaborer le concept de Circulation au sein des Trois Univers Discursifs de l'Indéterminé, de la Définition et du Dénivellement pour apprendre, penser et se construire.

Avec un peu d'audace, nous pourrions nous demander si ce concept de circulation au sein de trois Univers Discursifs, n'est pas la ZPD de Vygotski. Nous faisons cette proposition pour comprendre comment l'étayage peut aboutir à des apprentissages, pour rendre visibles les opérations cognitives à travers les observables langagiers qui signent des positions différentes au monde, à soi et à l'autre.

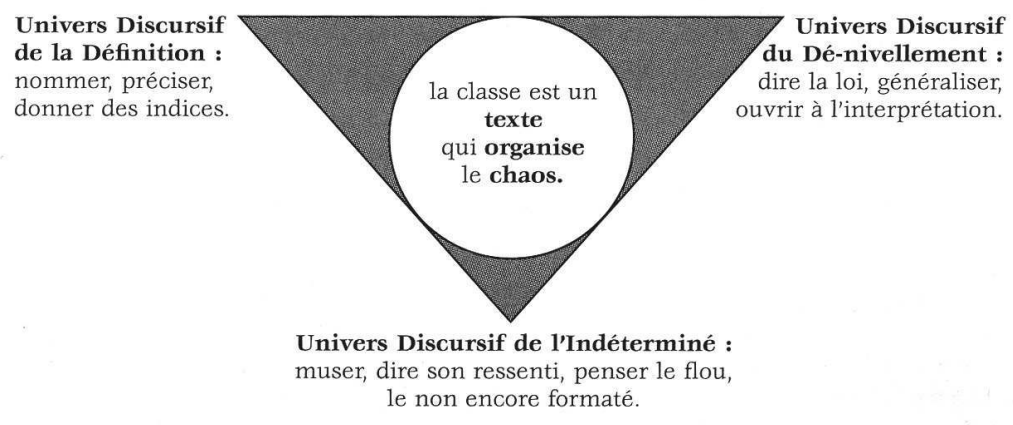

Schéma de la circulation au sein des trois univers discursifs

\section{BIBLIOGRAPHIE}

ADAM, J-M., 1984, Le récit. Paris : PUF. « Que sais-je?»

AUSTIN, J-L, 1970, Quand dire c'est faire. Paris : Le Seuil.

BAKHTINE, Mikhaël, 1984, Esthétique de la création verbale. Paris : Gallimard.

BALAT, Michel, 2000, Des fondements sémiotiques de la psychanalyse. Paris : L'Harmattan, 290.

BAUTIER, Elisabeth, CHARLOT, Bernard, ROCHEIX, Jean-Yves, 1993, Ecole et savoirs dans les banlieues, Paris, Armand Colin.

BAUTIER, Elisabeth, BUCHETON, Dominique, 1996, « Interactions : co-construction du sujet est des savoirs ", Le Français aujourd'hui, n¹13, Paris, AFEF, 24-32.

BRES, Jacques et Alii, 1999, L'autre en discours. Montpellier : Publications Université Paul-Valéry Montpellier III, 466.

BRONCKART, Jean-Paul, 2002, « La conscience comme analyseur des épistémologies de VYGOTSKI et PIAGET », Avec VYGOTSKI. Paris : La Dispute, 27-53.

BRUNER, Jérôme, 1991, Car la culture donne forme à l'esprit. Paris : Eshel.

BRUNER, Jérôme, 1997, Comment les enfants apprennent à parler. Paris : Retz.

BRUNER, Jérôme, 2002, Pourquoi nous racontons-nous des histoires ?. Paris : Retz, 112.

BUCHETON, Dominique, 1995, Ecriture réécritures. Récits d'adolescents. Neuchâtel : Peter Lang. 
BUCHETON, Dominique (Dir.), 1997, Conduites d'écriture au collège et au lycée professionnel, CRDP Versailles.

BUCHETON, Dominique, 2000, Ecriture et subjectivité, Mémoire pour l'habilitation à diriger des recherches. Montpellier : Université Montpellier III Paul-Valéry.

BUCHETON, Dominique (dir.), CHABANNE, Jean-Charles, et alii, 2002, Ecrire en ZEP un autre regard sur les écrits des élèves. Paris, DELAGRAVE, CRDP de Versailles, 191.

CHABANNE, Jean-Charles, BUCHETON, Dominique, 2002, Parler et écrire pour penser, apprendre et se construire. L'écrit et l'oral réflexifs. Paris : PUF, 252.

CLOT, Yves (dir.), 1999/2002, Avec VYGOTSKI, 2e édition augmentée. Paris : La Dispute, 345.

CYRULNIK, Boris, 1999, Un merveilleux malheur. Paris : Odile Jacob.

CYRULNIK, Boris, 1997/2001, L'ensorcellement du monde. Paris : Odile Jacob poches, 304.

CYRULNIK, Boris, 2003, Le murmure des fantômes. Paris : Odile Jacob, 259.

DECRON, Alain, 2001, L'élève Smahan : auteur d'une communauté sémiotique, mémoire de DEA.

Perpignan : Bibliothèque Universitaire.

DECRON, Alain, 2002, "L'élève-auteur : un sujet à la source d'une communauté sémiotique », Sémiotique peircienne : état des lieux. Perpignan : Presses Universitaires de Perpignan, 59-69.

DECRON, Alain, 2002, « Ecrits de travail dans une séquence scientifique en CE1 », Ecrire en ZEP un autre regard sur les écrits des élèves, ouvrage dirigé par Dominique BUCHETON. Paris : DELAGRAVE, CRDP de Versailles, 137-147.

FABRE, C., 1990, Les brouillons d'écoliers. Grenoble : CEDITEL.

FRANÇOIS, Frédéric et alii, 1990, La communication inégale. Lausanne : Delachaux et Niestlé, 276.

FRANÇOIS, Frédéric, 2002, « Mot et dialogue chez VYGOTSKI et BAKHTINE », Avec VYGOTSKI, sous la direction d'Yves Clot. Paris : La Dispute, 213-230.

JACQUES, F., 1979, Dialogiques. Paris : P.U.F.

JAPPY, Tony, RETHORE Joëlle (éditeurs), 2002, Sémiotique Peircienne : Etat des lieux. Perpignan :

Presses Universitaires de Perpignan, 175.

PEIRCE, Charles Sanders, 1978, Ecrits sur le signe, traduits et commentés par Gérard DELEDALLE. Paris : Le Seuil.

PEIRCE, Charles Sanders, 1993, A la recherche d'une méthode, sous la direction de Gérard DELEDALLE. Perpignan : Presses Universitaires de Perpignan, 371.

PERRAUDEAU, Michel, 1998, Echanger pour apprendre, l'entretien critique. Paris : Armand Colin, 159.

RETHORE, Joëlle, 1998, «L'entour du signe écrit : énonciation et lecture. De la nécessaire division du sujet ", La lecture, Degrés, Revue de synthèse à orientation sémiologique, n94, e1-e25.

REUTER, Y., 1986, « Lire, une pratique socioculturelle », Pratiques, 52.

REUTER, Y., 1989, « L'enseignement de l'écriture », Pratiques, 69.

REUTER, Y., 1996, Enseigner et apprendre à écrire. Paris : ESF.

RICCEUR, P., 1983, Temps et récit. Paris : Le Seuil.

ROCHEIX, Jean-Yves, 2002, « VYGOTSKI et WALLON : pour une pensée dialectique des rapports entre pensée et affect », Avec VYGOTSKI, sous la direction d'Yves CLOT. Paris : La Dispute, 121-140. 
SCHNEUWLY, B. et BRONCKART, J-P., 1985, VYGOTSKY aujourd'hui. Lausanne : Delachaux \& Niestle.

VANHULLE, S., 2002, « Comprendre des parcours d'écriture réflexive », Parler et écrire pour penser, apprendre et se construire. Paris : PUF, 227-245.

VION, Robert, 1992, La communication verbale. Analyse des interactions. Paris : Hachette Supérieur.

VYGOTSKI, Lev, 1934/1997, Pensée et langage, trad. de Françoise SEVE. Paris : La Dispute.

\section{RÉSUMÉS}

Cet article étudie le passage d'un enseignement à des apprentissages. Il met en évidence le rôle de la co-activité maître / élèves ainsi que la circulation de la parole au sein de trois univers discursifs différents qui signent des positions langagières et cognitives différentes face au monde pour apprendre, penser et se construire. Cette circulation discursive éclaire les enjeux et le fonctionnement de l'étayage à l'intérieur de la Zone Prochaine de Développement théorisée par VYGOTSKI (1934 / 1997).

This article studies the way from a teaching to acquiring knowledge. It reveals the role played by the co-activity between teacher and pupils and the circulation of speaking inside three different discursive universes which sign different linguistic and cognitive attitudes in front of the world to learn, think and build himself. This discursive circulation informs the stakes and the working of professional help inside the Proximal Development Zone theorized by Vygotski (1934 / 1997).

INDEX

Mots-clés : langage, univers discursif, co-activité, apprentissage, auteur

Keywords : language, discursive universe, co-activity, acquiring knowledge, author

\section{AUTEUR}

\section{ALAIN DECRON}

Doctorant en sciences du langage, Professeur de lettres, équipe LIRDEF-ALFA et ERT, IUFM

Montpellier 